\title{
AN ADDITIVE PROBLEM IN THE FOURIER COEFFICIENTS OF CUSP FORMS
}

\author{
GERGELY HARCOS
}

\begin{abstract}
We establish an estimate on sums of shifted products of Fourier coefficients coming from holomorphic or Maass cusp forms of arbitrary level and nebentypus. These sums are analogous to the binary additive divisor sum which has been studied extensively. As an application we derive, extending work of Duke, Friedlander and Iwaniec, a subconvex estimate on the critical line for $L$-functions associated to character twists of these cusp forms.
\end{abstract}

\section{Introduction AND StATEMEnt of RESUlts}

In the analytic theory of automorphic $L$-functions one often encounters sums of the form

$$
D_{f}(a, b ; h)=\sum_{a m \pm b n=h} \lambda_{\phi}(m) \lambda_{\psi}(n) f(a m, b n),
$$

where $a, b, h$ are positive integers, $\lambda_{\phi}(m)$ (resp. $\left.\lambda_{\psi}(n)\right)$ are the normalized Fourier coefficients of a holomorphic or Maass cusp form $\phi$ (resp. $\psi$ ) coming from an automorphic representation of $G L(2)$ over $\mathbb{Q}$ and $f$ is some nice weight function on $(0, \infty) \times(0, \infty)$. These sums have been studied extensively beginning with Selberg $\mathrm{Se}]$ (see also Good [G]) and are analogous to the generalized binary additive divisor sum where $\lambda_{\phi}(m)$ and $\lambda_{\psi}(n)$ are replaced by values of the divisor function:

$$
D_{f}^{\tau}(a, b ; h)=\sum_{a m \pm b n=h} \tau(m) \tau(n) f(a m, b n) .
$$

The analogy is deeper than formal, because $\tau(n)$ appears as the $n$th Fourier coefficient of the modular form $\left.\frac{\partial}{\partial s} E(z, s)\right|_{s=1 / 2}$ where $E(z, s)$ is the Eisenstein series for $S L_{2}(\mathbb{Z})$. In general one tries to deduce good estimates for these sums assuming the parameters $a, b, h$ are of considerable size.

The binary additive divisor problem has an extensive history and we refer the reader to D-F-I2 for a short introduction. Let us just mention that in the special case $a=b=1$ one can derive very sharp results by employing the spectral theory of automorphic forms for the full modular group [Mo]. This approach is hard to generalize for larger values of $a, b$ as one faces difficulties with small Laplacian eigenvalues for the congruence subgroup $\Gamma_{0}(a b)$. The idea of Duke, Friedlander and Iwaniec D-F-I2 is to combine the more elementary $\delta$-method (a variant of the classical circle method) with a Voronoi-type summation formula for the divisor function and then apply Weil's estimate for the individual Kloosterman sums

$$
S(m, n ; q)=\sum_{d(\bmod q)}^{*} e_{q}(d m+\bar{d} n)
$$

1991 Mathematics Subject Classification. Primary 11F30, 11F37; Secondary 11M41. 
that arise.

Assuming $a, b$ are coprime and the partial derivatives of the weight function $f$ satisfy the estimate

$$
x^{i} y^{j} f^{(i, j)}(x, y) \ll_{i, j}\left(1+\frac{x}{X}\right)^{-1}\left(1+\frac{y}{Y}\right)^{-1} P^{i+j}
$$

with some $P, X, Y \geq 1$ for all $i, j \geq 0$, they were able to deduce

$$
D_{f}^{\tau}(a, b ; h)=\int_{0}^{\infty} g(x, \pm x \mp h) d x+O\left(P^{5 / 4}(X+Y)^{1 / 4}(X Y)^{1 / 4+\epsilon}\right),
$$

where the implied constant depends on $\epsilon$ only,

$$
g(x, y)=f(x, y) \sum_{q=1}^{\infty} \frac{(a b, q)}{a b q^{2}} c_{q}(h)\left(\log x-\lambda_{a q}\right)\left(\log x-\lambda_{b q}\right),
$$

$c_{q}(h)=S(h, 0 ; q)$ denotes Ramanujan's sum and $\lambda_{a q}, \lambda_{b q}$ are constants given by

$$
\lambda_{a q}=2 \gamma+\log \frac{a q^{2}}{(a, q)^{2}} .
$$

As was pointed out in D-F-I2 the error term is smaller than the main term whenever

$$
a b \ll P^{-5 / 4}(X+Y)^{-5 / 4}(X Y)^{3 / 4-\epsilon} .
$$

The case $N=a=b=1$ of the sum $D_{f}(a, b ; h)$ has been discussed in detail via spectral theory by Jutila [J2, J33]. This approach is hard to generalize to levels $N>1$ even when $a=b=1$ because one faces with the difficulty of small Laplacian eigenvalues. A different spectral approach was developed by Sarnak for all levels. Using his estimates for triple products of eigenfunctions $\mathrm{S1}$ (see also $[\mathrm{P}, \mathrm{B}-\mathrm{R}$ ) he recently established quite strong bounds for $D_{f}(a, b ; h)$ at least when the shift parameter $h$ is relatively small [S2]. This method has the advantage of generalizing naturally to number fields $[\mathrm{P}-\mathrm{S}, \mathrm{C}-\mathrm{PS}-\mathrm{S}]$ but suffers from providing no information for large values of $h$. Our aim here is to establish, for all $h$, a nontrivial estimate on $D_{f}(a, b ; h)$ in the spirit of Duke, Friedlander and Iwaniec.

Theorem 1. Let $\lambda_{\phi}(m)$ (resp. $\lambda_{\psi}(n)$ ) be the normalized Fourier coefficients of a holomorphic or Maass cusp form $\phi$ (resp. $\psi$ ) of arbitrary level and nebentypus and suppose that $f$ satisfies (2). Then for coprime $a$ and $b$ we have

$$
D_{f}(a, b ; h) \ll P^{11 / 10}(a b)^{-1 / 10}(X+Y)^{1 / 10}(X Y)^{2 / 5+\epsilon},
$$

where the implied constant depends only on $\epsilon$ and the forms $\phi, \psi$.

See the next section for a precise definition of the notions in the theorem. We note that the theorem supercedes the trivial upper bound $(X Y / a b)^{1 / 2}$ (following from Cauchy's inequality) whenever

$$
a b \ll P^{-11 / 4}(X+Y)^{-1 / 4}(X Y)^{1 / 4-\epsilon} .
$$

As an application we prove a subconvex estimate on the critical line for $L$ functions associated to character twists of a fixed holomorphic or Maass cusp form $\phi$ of arbitrary level and nebentypus. We shall assume that $\phi$ is a primitive form, i.e. a newform in the sense of A-Z. Furthermore we normalize $\phi$ such that $\lambda_{\phi}(1)=1$ then $\lambda_{\phi}(m)(m \geq 1)$ defines a character of the corresponding Hecke algebra while $\lambda_{\phi}(-m)= \pm \lambda_{\phi}(m)$ (with a constant sign) when $\phi$ is a Maass form. In other words, $\phi$ defines a cuspidal automorphic representation of $G L(2)$ over $\mathbb{Q}$. The 
contragradient representation corresponds to the primitive cusp form $\tilde{\phi}(z)=\bar{\phi}(-\bar{z})$ with Fourier coefficients $\lambda_{\tilde{\phi}}(m)=\bar{\lambda}_{\phi}(m)$. If $q$ is an integer prime to the level and $\chi$ is a primitive Dirichlet character modulo $q$ then to the twisted primitive cusp form $\phi \otimes \chi$ is attached the $L$-function

$$
L(s, \phi \otimes \chi)=\sum_{m=1}^{\infty} \frac{\lambda_{\phi}(m) \chi(m)}{m^{s}}
$$

which is absolutely convergent for $\Re s>1$ and has an Euler product over the prime numbers. It has an analytic continuation to an entire function, as shown by Hecke, and satisfies a functional equation of the standard type. It follows from the Phragmén-Lindelöf convexity principle that for a fixed point on the critical line $\Re s=1 / 2$ we have a bound

$$
L(s, \phi \otimes \chi) \ll_{\epsilon, s, \phi} q^{1 / 2+\epsilon} .
$$

By a subconvexity estimate we mean one which replaces the convexity exponent $1 / 2$ by any smaller absolute constant. Upon the Generalized Riemann Hypothesis we would have the Generalized Lindelöf Hypothesis which asserts that any positive exponent is permissible. For the philosophy of breaking convexity in the analytic theory of $L$-functions and its importance for arithmetic we refer the reader to the excellent discussion by Iwaniec and Sarnak [I-S].

Theorem 2. Suppose that $\phi$ is a (normalized primitive) holomorphic or Maass cusp form of arbitrary level and nebentypus. Let $\Re s=1 / 2$ and $q$ be an integer prime to the level. If $\chi$ is a primitive Dirichlet character modulo $q$ then

$$
L(s, \phi \otimes \chi) \ll q^{13 / 27+\epsilon},
$$

where the implied constant depends only on $\epsilon, s$ and the form $\phi$.

This estimate (with the slightly better exponent 5/11) has been proved by Duke, Friedlander and Iwaniec D-F-I1] for the case of holomorphic forms $\phi$ of full level. They anticipated their method to be extendible to more general $L$-functions of rank one, and the present paper is indeed an extension of their work. Combining the estimate (4) at the central point $s=1 / 2$ with Waldsurger's theorem [Wa] (see also [K]) we get the bound

$$
c(q) \ll_{\epsilon} q^{13 / 54+\epsilon}, \quad q \text { square-free }
$$

for the Fourier coefficients of half-integral weight forms of arbitrary level. Such a nontrivial bound is the key step in the solution of the general ternary Linnik problem given by Duke and Schulze-Pillot D, D-SP].

The proof Theorem 1 is presented in Sections 2 through 5 and closely follows D-F-I2]. The heart of the argument is again a Voronoi-type formula (see Section 2) for transforming certain exponential sums defined by the coefficients $\lambda_{\phi}(m)$ and $\lambda_{\psi}(n)$ but this time the level of the forms imposes some restriction on the frequencies in the formula. As the $\delta$-method uses information at all frequencies (and in this sense it corresponds to the classical Farey dissection of the unit circle) we need to replace it (in Section 3) with another variant of the circle method (given by Jutila J1) which is more flexible in the choice of frequencies. After the transformations we shall encounter twisted Kloosterman sums

$$
S_{\chi}(m, n ; q)=\sum_{d(\bmod q)}^{*} \chi(d) e_{q}(d m+\bar{d} n),
$$


where $\chi$ is a Dirichlet character $\bmod q$. We shall make use of the usual WeilEstermann bound

$$
S_{\chi}(m, n ; q) \leq(m, n, q)^{1 / 2} q^{1 / 2} \tau(q)
$$

which holds true for these sums as well (the original proofs $[\mathrm{W}$, E] carry over with minor modifications).

Sections 6 through 8 are devoted to the proof of Theorem 2 . In Section 6 we reduce (4), via an approximate functional equation, to an inequality about certain finite sums involving about $q$ terms. In order to prove this inequality we use the amplification method which was introduced in [D-F-I1]. The idea is to choose suitable weights (called amplifiers) and to average with them the second moment of the finite sums arising from the family $\phi \otimes \chi$ of cusp forms ( $\chi$ varies, $\phi$ is fixed). We choose the amplifiers in such a way that one of the characters $\chi$ is emphasized while the average is still of moderate size. This forces, by positivity, $L(s, \phi \otimes \chi)$ to be small. In the course of evaluating the average of the second moments we encounter diagonal and off-diagonal terms and it is the off-diagonal contribution where Theorem 11 enters.

Acknowledgement. I am grateful to Peter Sarnak for calling my attention to this problem (a question originally raised by Atle Selberg, cf. [Se]).

\section{Summation formula for the Fourier coefficients}

We define the normalized Fourier coefficients of cusp forms as follows. Let $\phi(z)$ be a cusp form of level $N$ and nebentypus $\chi$, that is a holomorphic cusp form of some integral weight $k$ or a real-analytic Maass cusp form of some Laplacian eigenvalue $1 / 4+\mu^{2} \geq 0$. By definition, $\chi$ is a Dirichlet character $\bmod N$ and the form $\phi$ satisfies a transformation rule with respect to the Hecke congruence subgroup $\Gamma_{0}(N)$

$$
\phi \circ[\gamma]=\chi(d) u, \quad \gamma=\left(\begin{array}{ll}
a & b \\
c & d
\end{array}\right) \in \Gamma_{0}(N),
$$

where

$$
\phi \circ[\gamma](z)= \begin{cases}\phi(\gamma z)(c z+d)^{-k} & \text { if } \phi \text { is holomorphic, } \\ \phi(\gamma z) & \text { if } \phi \text { is real-analytic, }\end{cases}
$$

and $\Gamma_{0}(N)$ acts on the upper half-plane $\mathcal{H}=\{z: \Im z>0\}$ by fractional linear transformations. Also, the form $\phi$ is holomorphic or real-analytic on $\mathcal{H}$ and decays exponentially to zero at each cusp. Any such $\phi$ admits the Fourier expansion

$$
\phi(z)=\sum_{m \neq 0} \hat{\lambda}_{\phi}(m) W(m z),
$$

where

$$
W(z)= \begin{cases}e(z) & \text { if } \phi \text { is holomorphic, } \\ W_{1 / 2+i \mu}=2|y|^{1 / 2} K_{i \mu}(2 \pi|y|) e(x) & \text { if } \phi \text { is real-analytic. }\end{cases}
$$

Here $e(z)=e^{2 \pi i z}, z=x+i y$ and $K_{i \mu}$ is the Macdonald-Bessel function. If $\phi$ is holomorphic, $\hat{\lambda}_{\phi}(m)$ vanishes for $m \leq 0$. We define the normalized Fourier 
coefficients of $\phi$ as

$$
\lambda_{\phi}(m)= \begin{cases}\hat{\lambda}_{\phi}(m) m^{\frac{1-k}{2}} & \text { if } \phi \text { is holomorphic, } \\ \hat{\lambda}_{\phi}(m)|m|^{\frac{1}{2}} & \text { if } \phi \text { is real-analytic. }\end{cases}
$$

This normalization corresponds to the Ramanujan Conjecture which asserts that

$$
\lambda_{\phi}(m) \ll_{\epsilon, \phi} m^{\epsilon} .
$$

Rankin-Selberg theory implies that the conjecture holds on average in the form

$$
\sum_{1 \leq m \leq x}\left|\lambda_{\phi}(m)\right|^{2} \ll_{\phi} x
$$

Various Voronoi-type summation formulas are fulfilled by these coefficients. In the case of full level $(N=1)$ Duke and Iwaniec D-I established such a formula for holomorphic cusp forms and Meurman [M] for Maass cusp forms. These can be generalized to arbitrary level and nebentypus with obvious minor modifications as follows.

Proposition 1. Let $d$ and $q$ be coprime integers such that $N \mid q$, and let $g$ be a smooth, compactly supported function on $(0, \infty)$. If $\phi$ is a holomorphic cusp form of level $N$, nebentypus $\chi$ and integral weight $k$ then

$$
\chi(d) \sum_{n=1}^{\infty} \lambda_{\phi}(m) e_{q}(d m) g(m)=\sum_{m=1}^{\infty} \lambda_{\phi}(m) e_{q}(-\bar{d} m) \hat{g}(m),
$$

where

$$
\hat{g}(y)=\frac{2 \pi i^{k}}{q} \int_{0}^{\infty} g(x) J_{k-1}\left(\frac{4 \pi \sqrt{x y}}{q}\right) d x .
$$

If $\phi$ is a real-analytic Maass cusp form of level $N$, nebentypus $\chi$ and Laplacian eigenvalue $1 / 4+\mu^{2} \geq 0$ then

$$
\chi(d) \sum_{m=1}^{\infty} \lambda_{\phi}(m) e_{q}(d m) g(m)=\sum_{ \pm} \sum_{m=1}^{\infty} \lambda_{\phi}(\mp m) e_{q}( \pm \bar{d} m) g^{ \pm}(m),
$$

where

$$
\begin{aligned}
& g^{-}(y)=-\frac{\pi}{q \cosh \pi \mu} \int_{0}^{\infty} g(x)\left\{Y_{2 i \mu}+Y_{-2 i \mu}\right\}\left(\frac{4 \pi \sqrt{x y}}{q}\right) d x, \\
& g^{+}(y)=\frac{4 \cosh \pi \mu}{q} \int_{0}^{\infty} g(x) K_{2 i \mu}\left(\frac{4 \pi \sqrt{x y}}{q}\right) d x .
\end{aligned}
$$

Here $\bar{d}$ is a multiplicative inverse of $d \bmod q, e_{q}(x)=e(x / q)=e^{2 \pi i x / q}$ and $J_{k-1}$, $Y_{ \pm 2 i \mu}, K_{2 i \mu}$ are Bessel functions.

The proof for the holomorphic case $[\mathrm{D}-\mathrm{I}]$ is a straightforward application of Laplace transforms. Meurman's proof for the real-analytic case [M] is more involved, but only because he considers a wider class of test functions $g$ and has to deal with delicate convergence issues. For smooth, compactly supported functions $g$ as in our formulation these difficulties do not arise and one can give a much simpler proof based on Mellin transformation, the functional equations of the $L$-series attached to additive twists of $\phi$ (see [M] ), and Barnes' formulas for the gamma function. Indeed, Lemma 5 in [St] (a special case of Meurman's summation formula) has been proved by such an approach. We expressed the formula for the 
non-holomorphic case in terms of $K$ and $Y$ Bessel functions in order to emphasize the analogy with the Voronoi-type formula for the divisor function (where one has $\mu=0$ ) as derived by Jutila J4, J5.

\section{Setting Up the CirCle Method}

For sake of exposition we shall only present the case of real-analytic Maass forms and the equation $a m-b n=h$. The other cases follow along the same lines by changing Bessel functions and signs at relevant places of the argument. In our inequalities $\epsilon$ will always denote a small positive number whose actual value is allowed to change at each occurence. Furthermore, unless otherwise indicated, implied constants will depend on $\epsilon$ and the cusp forms only (including dependence on the level, nebentypus characters and Laplacian eigenvalues).

Let $\phi(z)$ (resp. $\psi(z)$ ) be a Maass cusp form of level $N$, nebentypus $\chi$ (resp. $\omega$ ) and Laplacian eigenvalue $1 / 4+\mu^{2} \geq 0$ (resp. $1 / 4+\nu^{2} \geq 0$ ) whose normalized Fourier coefficients are $\lambda_{\phi}(m)$ (resp. $\lambda_{\psi}(n)$ ), i.e.,

$$
\begin{aligned}
\phi(x+i y) & =\sqrt{y} \sum_{m \neq 0} \lambda_{\phi}(m) K_{i \mu}(2 \pi|m| y) e(m x), \\
\psi(x+i y) & =\sqrt{y} \sum_{n \neq 0} \lambda_{\psi}(n) K_{i \nu}(2 \pi|n| y) e(n x) .
\end{aligned}
$$

Using a smooth partition of unity for the proof of Theorem 1 we may assume that $f(x, y)$ is supported in the box $[X, 2 X] \times[Y, 2 Y]$. Then we can also assume that

$$
h \leq 2(X+Y)
$$

for otherwise $D_{f}(a, b ; h)$ vanishes trivially. We shall attach, as in D-F-I2, a redundant factor $g(x-y-h)$ to $f(x, y)$ where $g(t)$ is a smooth function supported on $|t|<\delta^{-1}$ such that $g(0)=1$ and $g^{(i)} \ll_{i} \delta^{i}$. This, of course, does not alter $D_{f}(a, b ; h)$. We choose

$$
\delta=P \frac{X+Y}{X Y}
$$

so that, by (2), the new function

$$
F(x, y)=f(x, y) g(x-y-h)
$$

has partial derivatives bounded by

$$
F^{(i, j)} \ll_{i, j} \delta^{i+j}
$$

We apply the Hardy-Littlewood method to detect the equation $a m-b n=h$, that is, we express $D_{F}(a, b ; h)$ as the integral of a certain exponential sum over the unit interval $[0,1]$. We get

$$
D_{f}(a, b ; h)=D_{F}(a, b ; h)=\int_{0}^{1} G(\alpha) d \alpha,
$$

where

$$
G(\alpha)=\sum_{m, n} \lambda_{\phi}(m) \lambda_{\psi}(n) F(a m, b n) e((a m-b n-h) \alpha) .
$$

We shall approximate this integral by the following proposition of Jutila (a consequence of the main theorem in [J1]) 
Proposition 2 (Jutila). Let $\mathcal{Q}$ be a nonempty set of integers $Q \leq q \leq 2 Q$ where $Q \geq 1$. Let $Q^{-2} \leq \delta \leq Q^{-1}$ and for each fraction $d / q$ (in its lowest terms) denote by $I_{d / q}(\alpha)$ the characteristic function of the interval $[d / q-\delta, d / q+\delta]$. Write $L$ for the number of such intervals, i.e.,

$$
L=\sum_{q \in \mathcal{Q}} \varphi(q)
$$

and put

$$
\tilde{I}(\alpha)=\frac{1}{2 \delta L} \sum_{q \in \mathcal{Q}} \sum_{d(\bmod q)}^{*} I_{d / q}(\alpha)
$$

If $I(\alpha)$ is the characteristic function of the unit interval $[0,1]$ then

$$
\int_{-\infty}^{\infty}(I(\alpha)-\tilde{I}(\alpha))^{2} d x \ll \delta^{-1} L^{-2} Q^{2+\epsilon}
$$

where the implied constant depends on $\epsilon$ only.

We shall choose some $Q$ and apply the proposition with a set of denominators of the form

$$
\mathcal{Q}=\{q \in[Q, 2 Q]: N a b \mid q \text { and }(h, q)=(h, N a b)\} .
$$

By a result of Jacobsthal $\mathrm{Ja}$ the largest gap between reduced residue classes mod $h$ is of size $\ll h^{\epsilon}$, whence, by (耳),

$$
|\mathcal{Q}| \gg \frac{Q(X Y)^{-\epsilon}}{a b}
$$

assuming the right hand side exceeds some positive constant $c=c(\epsilon, N)$. Moreover, we shall assume that

$$
Q^{-2} \leq \delta \leq Q^{-1}
$$

so that also

$$
1 \leq Q \leq X Y
$$

whence (10) yields

$$
L \gg \frac{Q^{2}(X Y)^{-\epsilon}}{a b}
$$

We clearly have

$$
D_{F}(a, b ; h)-\tilde{D}_{F}(a, b ; h) \leq\|G\|_{\infty}\|I-\tilde{I}\|_{1},
$$

where

$$
\begin{aligned}
\tilde{D}_{F}(a, b ; h) & =\int_{-\infty}^{\infty} G(\alpha) \tilde{I}(\alpha) d \alpha=\frac{1}{2 \delta L} \sum_{q \in \mathcal{Q}} \sum_{d(\bmod q)}^{*} \int_{-\infty}^{\infty} G(\alpha) I_{d / q}(\alpha) d \alpha \\
& =\frac{1}{2 \delta L} \sum_{q \in \mathcal{Q}} \sum_{d(\bmod q)}^{*} \int_{-\delta}^{\delta} G(d / q+\beta) d \beta=\frac{1}{2 \delta L} \sum_{q \in \mathcal{Q}} \sum_{d(\bmod q)}^{*} \mathfrak{I}_{d / q},
\end{aligned}
$$

say. To derive an upper estimate for $G(\alpha)$ we express it as

$$
G(\alpha)=\int_{0}^{\infty} \int_{0}^{\infty} F(x, y) e(-h \alpha) d S(x / a) d T(y / b)
$$


where

$$
S(x)=\sum_{1 \leq m \leq x} \lambda_{\phi}(m) e(a m \alpha), \quad T(y)=\sum_{1 \leq n \leq y} \lambda_{\psi}(n) e(-b n \alpha) .
$$

Then, integrating by parts,

$$
G(\alpha)=\int_{0}^{\infty} \int_{0}^{\infty} F^{(1,1)}(x, y) e(-h \alpha) S(x / a) T(y / b) d x d y
$$

therefore (9) combined with Wilton's classical estimate

$$
S(x) \ll x^{1 / 2} \log x, \quad T(y) \ll y^{1 / 2} \log y
$$

yields

$$
\|G\|_{\infty} \ll \frac{(X Y)^{1 / 2+\epsilon}}{(a b)^{1 / 2}}\left\|F^{(1,1)}\right\|_{1} \ll \frac{\delta(X Y)^{3 / 2+\epsilon}}{(a b)^{1 / 2}(X+Y)} .
$$

Also, by (13) and Proposition 2 we get

$$
\|I-\tilde{I}\|_{1} \leq 3\|I-\tilde{I}\|_{2} \ll \frac{a b(X Y)^{\epsilon}}{\delta^{1 / 2} Q},
$$

so that (14) becomes

$$
D_{F}(a, b ; h)-\tilde{D}_{F}(a, b ; h) \ll \frac{(a b)^{1 / 2} \delta^{1 / 2}}{Q} \cdot \frac{(X Y)^{3 / 2+\epsilon}}{(X+Y)} .
$$

\section{TRANSFORMing EXPONENTIAL SUMS}

The contribution of the interval $[d / q-\delta, d / q+\delta]$ can be expressed as

$$
\mathfrak{I}_{d / q}=\int_{-\delta}^{\delta} G(d / q+\beta) d \beta=e_{q}(-d h) \sum_{m, n} \lambda_{\phi}(m) \lambda_{\psi}(n) e_{q}(d(a m-b n)) E(m, n),
$$

where

$$
E(x, y)=F(a x, b y) \int_{-\delta}^{\delta} e((a x-b y-h) \beta) d \beta .
$$

Using (9) we clearly have

$$
E^{(i, j)} \ll_{i, j} \delta^{i+j+1} a^{i} b^{j},
$$

and we also record, for further reference, that

$$
\left\|E^{(i, j)}\right\|_{1} \ll_{i, j} \delta^{i+j} a^{i-1} b^{j-1} \frac{X Y}{X+Y} .
$$

We assume that $q \in \mathcal{Q}$, hence $N a b \mid q$ and we can apply Proposition 1 to yield

$$
\mathfrak{I}_{d / q}=\overline{\chi \omega}(d) e_{q}(-d h) \sum_{ \pm \pm} \sum_{m, n \geq 1} \lambda_{\phi}(\mp m) \lambda_{\psi}(\mp n) e_{q}(\bar{d}( \pm a m \mp b n)) E^{ \pm \pm}(m, n),
$$

where the corresponding signs must be matched and

$$
\begin{gathered}
E^{ \pm \pm}(m, n)=\frac{a b}{q^{2}} \int_{0}^{\infty} \int_{0}^{\infty} E(x, y) M_{2 i \mu}^{ \pm}\left(\frac{4 \pi a \sqrt{m x}}{q}\right) M_{2 i \nu}^{ \pm}\left(\frac{4 \pi b \sqrt{n y}}{q}\right) d x d y \\
M_{2 i r}^{+}=(4 \cosh \pi r) K_{2 i r}, \quad M_{2 i r}^{-}=-\frac{\pi}{\cosh \pi r}\left\{Y_{2 i r}+Y_{-2 i r}\right\}
\end{gathered}
$$


By summing over the residue classes we get

$$
\sum_{d(\bmod q)}^{*} \mathfrak{I}_{d / q}=\sum_{ \pm \pm} \sum_{m, n \geq 1} \lambda_{\phi}(\mp m) \lambda_{\psi}(\mp n) S_{\overline{\chi \omega}}(-h, \pm a m \mp b n ; q) E^{ \pm \pm}(m, n) .
$$

In order to estimate the twisted Kloosterman sum we observe that the greatest common divisor $(-h, \pm a m \mp b n, q)$ divides $N(h, n, a)(h, m, b)$ as follows from the relations $(a, b)=1$ and $(h, q)=(h, N a b)$, therefore (5) and (12) imply that

$$
S_{\overline{\chi \omega}}(-h, \pm a m \mp b n ; q) \ll(h, m)^{1 / 2}(h, n)^{1 / 2} Q^{1 / 2}(X Y)^{\epsilon} .
$$

We estimate $E^{ \pm \pm}(m, n)$ by successive applications of integration by parts and the relations

$$
\begin{gathered}
\frac{d}{d z}\left(z^{s} K_{s}(z)\right)=-z^{s} K_{s-1}(z), \quad \frac{d}{d z}\left(z^{s} Y_{s}(z)\right)=z^{s} Y_{s-1}(z) ; \\
K_{s}(z) \ll_{s} z^{-1 / 2}, \quad Y_{s}(z) \ll_{s} z^{-1 / 2}, \quad z>0 .
\end{gathered}
$$

We get, for any integers $i, j \geq 0$,

$$
\begin{aligned}
E^{ \pm \pm}(m, n) \ll_{i, j} & \frac{a b}{Q^{2}}\left(\frac{Q}{a \sqrt{m}}\right)^{i+\frac{1}{2}}\left(\frac{Q}{b \sqrt{n}}\right)^{j+\frac{1}{2}} \\
& \times \max _{\substack{0 \leq k \leq i \\
0 \leq l \leq j}}\left(\frac{X}{a}\right)^{k-\frac{i}{2}-\frac{1}{4}}\left(\frac{Y}{b}\right)^{l-\frac{j}{2}-\frac{1}{4}}\left\|E^{(k, l)}\right\|_{1},
\end{aligned}
$$

i.e., by 16$)$,

$$
\begin{aligned}
E^{ \pm \pm}(m, n) \ll_{i, j} & \frac{1}{Q^{2}}\left(\frac{Q}{a \sqrt{m}}\right)^{i+\frac{1}{2}}\left(\frac{Q}{b \sqrt{n}}\right)^{j+\frac{1}{2}} \\
& \times\left(\frac{X}{a}\right)^{-\frac{i}{2}-\frac{1}{4}}\left(\frac{Y}{b}\right)^{-\frac{j}{2}-\frac{1}{4}} \frac{X Y}{X+Y} \max _{\substack{0 \leq k \leq i \\
0 \leq l \leq j}}(X \delta)^{k}(Y \delta)^{l} .
\end{aligned}
$$

Therefore

$$
E^{ \pm \pm}(m, n) \ll_{i, j} \frac{(X Y)^{1 / 2}}{\delta Q^{2}(X+Y)}\left(\frac{(\delta Q)^{2} X}{a m}\right)^{\frac{i}{2}+\frac{1}{4}}\left(\frac{(\delta Q)^{2} Y}{b n}\right)^{\frac{j}{2}+\frac{1}{4}},
$$

suggesting that we can neglect the contribution to (17) of those pairs $(m, n)$ for which $a m / X$ or $b n / Y$ is $>(\delta Q)^{2}(X Y)^{\epsilon}$. Indeed, if we apply (6) to $\phi$ and $\psi$ to see that

$$
\sum_{1 \leq m \leq x}\left|\lambda_{\phi}(\mp m)\right|(h, m)^{1 / 2} \ll x \tau^{1 / 2}(h), \quad \sum_{1 \leq n \leq y}\left|\lambda_{\psi}(\mp n)\right|(h, n)^{1 / 2} \ll y \tau^{1 / 2}(h)
$$

then we can specify $i$ and $j$ large enough (in terms of $\epsilon$ ) to deduce from (18) and (19) that the contribution to (17) of those terms with $m$ or $n$ large is

$$
\ll \tau(h) \frac{\delta^{3} Q^{5 / 2}(X Y)^{-100}}{a b(X+Y)},
$$

say, while the choice $i=j=0$ in 19 shows that the remaining terms (for which $a m / X$ and $b n / Y$ are at most $\left.(\delta Q)^{2}(X Y)^{\epsilon}\right)$ contribute

$$
\ll \tau(h) \frac{\delta^{3} Q^{5 / 2}(X Y)^{3 / 2+\epsilon}}{a b(X+Y)} .
$$


Hence, by (13),

$$
\tilde{D}_{F}(a, b ; h)=\frac{1}{2 \delta L} \sum_{q \in \mathcal{Q}} \sum_{d(\bmod q)}^{*} \mathfrak{I}_{d / q} \ll \frac{\delta^{2} Q^{3 / 2}}{a b} \cdot \frac{(X Y)^{3 / 2+\epsilon}}{(X+Y)} .
$$

\section{Concluding Theorem 1}

The inequalities (15) and (20) show that the optimal balance is achieved when

$$
\delta^{3} Q^{5}(a b)^{-3} \asymp 1 .
$$

A natural choice is given by

$$
\delta^{3} Q^{5}=(c a b)^{3},
$$

where $c$ is the constant appearing in the remark after (10). This choice provides the theorem whenever the conditions of Proposition 2 are satisfied, that is, when $Q \geq \operatorname{cab}(X Y)^{\epsilon}$ and (11) hold simultaneously. It turns out that this is the case whenever

$$
c a b \leq P^{-2 / 3}(X+Y)^{-2 / 3}(X Y)^{2 / 3-\epsilon},
$$

in particular, whenever (3) is true. However, when (3) fails, Theorem 11 follows from the Cauchy bound $(X Y / a b)^{1 / 2}$, as we already pointed out in the introduction. The proof of Theorem 1 is complete.

\section{Approximate functional EQUATion}

Let $\phi$ be a normalized primitive holomorphic or Maass cusp form of arbitrary level and nebentypus, $\Re s=1 / 2$, and $\chi$ a primitive character modulo $q$ where $q$ is prime to the level. Using the functional equation of the $L$-function attached to the twisted primitive cusp form $\phi \otimes \chi$ and a standard technique involving Mellin transforms we can express the special value $L(s, \phi \otimes \chi)$ as a sum of two Dirichlet series of essentially $\sqrt{C}$ terms where $C=C(s, \phi \otimes \chi)$ is the analytic conductor defined by $[\mathrm{I}-\mathrm{S}]$. More precisely, $C \asymp q^{2}$ where the implied constants depend only on $s$ and $\phi$, therefore a special case of Theorem 1 in $[\mathrm{H}]$ gives the following

Proposition 3. There is a smooth function $f:(0, \infty) \rightarrow \mathbb{C}$ and a complex number $\lambda$ of modulus 1 such that

$$
L(s, \phi \otimes \chi)=\sum_{m=1}^{\infty} \frac{\lambda_{\phi}(m) \chi(m)}{m^{1 / 2}} f\left(\frac{m}{q}\right)+\lambda \sum_{m=1}^{\infty} \frac{\bar{\lambda}_{\phi}(m) \bar{\chi}(m)}{m^{1 / 2}} \bar{f}\left(\frac{m}{q}\right) .
$$

The function $f$ and its partial derivatives $f^{(j)}(j=1,2, \ldots)$ satisfy the following uniform growth estimates at 0 and infinity:

$$
\begin{gathered}
f(x)= \begin{cases}1+O\left(x^{\sigma}\right), & 0<\sigma<1 / 5 ; \\
O\left(x^{-\sigma}\right), & \sigma>0 .\end{cases} \\
f^{(j)}(x) \ll x^{-\sigma}, \quad \sigma>j-1 / 5 .
\end{gathered}
$$

The implied constants depend only on $\sigma, j, s$ and the form $\phi$. 
For any positive numbers $A$ and $\epsilon$ we obtain, using (6), an expression

$$
L(s, \phi \otimes \chi)=T+\lambda \bar{T}+O_{A, \epsilon, s, \phi}\left(q^{-A}\right),
$$

where

$$
T=\sum_{1 \leq m \leq q^{1+\epsilon}} \frac{\lambda_{\phi}(m) \chi(m) g(m)}{m^{1 / 2}},
$$

and $g:(0, \infty) \rightarrow \mathbb{C}$ is a smooth function satisfying

$$
g^{(j)}(x) \ll_{j, s, \phi} x^{-j}
$$

Therefore, applying partial summation and a smooth dyadic decomposition we can reduce Theorem 2 to the following

Proposition 4. Let $1 \leq M \leq q^{1+\epsilon}$ and $h$ be a smooth function supported in $[M, 2 M]$ such that $h^{(j)} \ll_{j} M^{-\bar{j}}$. Then

$$
\sum_{m=1}^{\infty} \lambda_{\phi}(m) \chi(m) h(m) \ll q^{17 / 54+\epsilon} M^{2 / 3},
$$

where the implied constant depends only on $\epsilon$ and the form $\phi$.

\section{AmplificAtion}

Our purpose is to prove Proposition 4 As in D-F-I1 we shall estimate from both ways the amplified sum

$$
S=\sum_{\omega \bmod q}^{*}\left|\sum_{1 \leq l \leq L} \bar{\chi}(l) \omega(l)\right|^{2}\left|S_{\omega}\right|^{2},
$$

where $\omega$ runs through the primitive characters modulo $q, L$ is a parameter to be chosen later in terms of $M$ and $q$, and

$$
S_{\omega}=\sum_{m=1}^{\infty} \lambda_{\phi}(m) \omega(m) h(m)
$$

Assuming $L \geq c(\epsilon) q^{\epsilon}$ (indeed this will be the case whenever $\epsilon<1 / 27$, cf. (25)) it follows, using the result of Jacobsthal [Ja] that the largest gap between reduced residue classes $\bmod q$ is of size $\ll q^{\epsilon}$, that

$$
S \gg q^{-\epsilon} L^{2}\left|S_{\chi}\right|^{2} .
$$

On the other hand, expanding each primitive $\omega$ in $S$ using Gauss sums and then extending the resulting summation to all characters $\bmod q$, we get by orthogonality,

$$
S \leq \frac{\varphi(q)}{q} \sum_{d(\bmod q)}^{*}\left|\sum_{n} a(n) e_{q}(d n)\right|^{2},
$$

where

$$
a(n)=\sum_{\substack{l m=n \\ 1 \leq l \leq L}} \bar{\chi}(l) \lambda_{\phi}(m) h(m) .
$$


It is clear that the coefficients $a(n)$ are supported in the interval $[1, N]$ where $N=2 L M$. Extending the summation to all residue classes $d$ the previous inequality becomes

$$
S \leq \varphi(q) \sum_{h \equiv 0(\bmod q)} D(h),
$$

where

$$
D(h)=\sum_{n_{1}-n_{2}=h} a\left(n_{1}\right) \bar{a}\left(n_{2}\right) .
$$

Using the Rankin-Selberg bound (6) it is simple to estimate the diagonal contribution $D(0)$. Indeed, by $h \ll 1$ we get

$$
\begin{aligned}
D(0) & =\sum_{n}|a(n)|^{2} \ll \sum_{\substack{l_{1} m_{1}=l_{2} m_{2} \\
1 \leq l_{1}, l_{2} \leq L \\
M \leq m_{1}, m_{2} \leq 2 M}} \lambda_{\phi}\left(m_{1}\right) \bar{\lambda}_{\phi}\left(m_{2}\right) \\
& \ll \sum_{\substack{1 \leq l \leq L \\
M \leq m \leq 2 M}}\left|\lambda_{\phi}(m)\right|^{2} \tau(m l) \ll N^{\epsilon} L \sum_{M \leq m \leq 2 M}\left|\lambda_{\phi}(m)\right|^{2},
\end{aligned}
$$

whence

$$
D(0)=\sum_{n}|a(n)|^{2} \ll N^{1+\epsilon} .
$$

In order to estimate the non-diagonal terms $D(h)(h \neq 0)$ we shall refer to Theorem 1. Clearly, we can rewrite each term as

$$
D(h)=\sum_{1 \leq l_{1}, l_{2} \leq L} \bar{\chi}\left(l_{1}\right) \chi\left(l_{2}\right) \sum_{l_{1} m_{1}-l_{2} m_{2}=h} \lambda_{\phi}\left(m_{1}\right) \bar{\lambda}_{\phi}\left(m_{2}\right) h\left(m_{1}\right) \bar{h}\left(m_{2}\right) .
$$

The inner sum is of type (11), because $\bar{\lambda}_{\phi}(m)$ is just the $m$-th normalized Fourier coefficient of the contragradient cusp form $\tilde{\phi}(z)=\bar{\phi}(-\bar{z})$. For each pair $\left(l_{1}, l_{2}\right)$ we shall apply Theorem 11 with $a=l_{1} /\left(l_{1}, l_{2}\right), b=l_{2} /\left(l_{1}, l_{2}\right), X=a M$ and $Y=b M$ to conclude that

$$
D(h) \ll L^{2}(a+b)^{1 / 10}(a b)^{3 / 10+\epsilon} M^{9 / 10+\epsilon} \ll L^{27 / 10+\epsilon} M^{9 / 10+\epsilon} .
$$

\section{Concluding Theorem}

Inserting the bounds (23) and (24) into (22) it follows that

$$
S \ll N^{\epsilon} \varphi(q)\left(N+\frac{N}{q} L^{27 / 10} M^{9 / 10}\right) .
$$

This shows that the optimal choice for $L$ is provided by

$$
q=L^{27 / 10} M^{9 / 10}
$$

whence (21) yields

$$
S_{\chi} \ll q^{\epsilon} L^{-1}|S|^{1 / 2} \ll(q N)^{1 / 2+\epsilon} L^{-1} \ll(q M / L)^{1 / 2+\epsilon} .
$$

Substituting (25) we get

$$
S_{\chi} \ll\left(q M q^{-10 / 27} M^{1 / 3}\right)^{1 / 2+\epsilon} \ll q^{17 / 54+\epsilon} M^{2 / 3}
$$

which is precisely the conclusion of Proposition t The proof of Theorem 2 is complete. 


\section{REFERENCES}

[A-L] Atkin, A.O.L.-Lehner, J., Hecke operators on $\Gamma_{0}(m)$, Math. Ann. 185 (1970), 134-160.

[B-R] Bernstein, J.-Reznikov, A., Analytic continuation of representations and estimates of automorphic forms, Ann. of Math. 150 (1999), 329-352.

[C-PS-S] Cogdell, J.W.-Piatetski-Shapiro, I.I.-Sarnak, P., Estimates for Hilbert modular Lfunctions and applications, in preparation

[D] Duke, W., Hyperbolic distribution problems and half-integral weight Maass forms, Invent. Math. 92 (1988), 73-90.

[D-F-I1] Duke, W.-Friedlander, J.B.-Iwaniec, H., Bounds for automorphic L-functions, Invent. Math. 112 (1993), 1-8.

[D-F-I2] Duke, W.-Friedlander, J.B.-Iwaniec, H., A quadratic divisor problem, Invent. Math. 115 (1994), 209-217.

[D-I] Duke, W.-Iwaniec, H., Bilinear forms in the Fourier coefficients of half-integral weight cusp forms and sums over primes, Math. Ann. 286 (1990), 783-802.

[D-SP] Duke, W.-Schulze-Pillot, R., Representations of integers by positive ternary quadratic forms and equidistribution of lattice points on ellipsoids, Invent. Math. 99 (1990), 49-57.

[E] $\quad$ Estermann, T., On Kloosterman's sum, Mathematika 8 (1961), 83-86.

[G] Good, A., The square mean of a Dirichlet series associated with cusp forms, Mathematika 29 (1982), 278-295.

$[\mathrm{H}] \quad$ Harcos, G., Uniform approximate functional equation for principal $L$-functions, preprint (2001)

[I-S] Iwaniec, H.-Sarnak, P., Perspectives on the analytic theory of $L$-functions, Geom. Funct. Anal. Special Volume (2000), 705-741.

[Ja] Jacobsthal, E., Über Sequenzen ganzer Zahlen, von denen keine zu $n$ teilerfremd ist. I-III. (German), Norke Vid. Selsk. Forh. Trondheim 33 (1961), 117-139.

[J1] Jutila, M., Transformations of exponential sums, Proceedings of the Amalfi Conference on Analytic Number Theory (Maiori, 1989), 263-270, Univ. Salerno, Salerno (1992)

[J2] Jutila, M., The additive divisor problem and its analogs for Fourier coefficients of cusp forms. I., Math. Z. 223 (1996), 435-461.; II., Math. Z. 225 (1997), 625-637.

[J3] Jutila, M., Convolutions of Fourier coefficients of cusp forms, Publ. Inst. Math. (Beograd) (N.S.) 65(79) (1999), 31-51.

[J4] Jutila, M., On exponential sums involving the divisor function, J. Reine Angew. Math. 355 (1985), 173-190.

[J5] Jutila, M., A method in the theory of exponential sums, Tata Lect. Notes Math. 80, Bombay (1987)

[K] Kohnen, W., Fourier coefficients of modular forms of half-integral weight, Math. Ann. 271 (1985), 237-268.

[M] Meurman, T., On exponential sums involving the Fourier coefficients of Maass wave forms, J. Reine Angew. Math. 384 (1988), 192-207.

[Mo] Motohashi, Y., The binary additive divisor problem, Ann. Sci. École Norm. Sup. 27 (1994), 529-572.

[P] Petridis, Y., On squares of eigenfuncions for the hyperbolic plane and a new bound on certain L-series, Internat. Math. Res. Notices (1995), 111-127.

[P-S] Petridis, Y.-Sarnak, P., Quantum unique ergodicity for $S L_{2}(\mathcal{O}) \backslash \mathbf{H}^{3}$ and estimates for $L$-functions, J. Evol. Equ., to appear

[S1] Sarnak, P., Integrals of products of eigenfunctions, Internat. Math. Res. Notices (1994), 251-260.

[S2] Sarnak, P., Estimates for Rankin-Selberg $L$-functions and quantum unique ergodicity, preprint (2001)

[Se] Selberg, A., On the estimation of Fourier coefficients of modular forms, Proc. Symp. Pure Math. 7 (1965), 1-15.

[St] Stefanicki, T., Non-vanishing of $L$-functions attached to automorphic representations of $G L(2)$ over Q, J. Reine Angew. Math. 474 (1996), 1-24.

[Wa] Waldspurger, J.-L., Sur les coefficients de Fourier des formes modulaires de poids demientier, Math. Pures Appl. 60 (1981), 375-484.

[W] Weil, A., On some exponential sums, Proc. Nat. Acad. Sci. U. S. A. 34 (1948), $204-207$. 
Department of Mathematics, Princeton University, Fine Hall, Washington Road, Princeton, NJ 08544, USA

E-mail address: gharcos@math.princeton.edu 